\title{
La modalidad de agentes multiplicadores para orientar a madres y padres de familia
}

\section{Multiplier Modality in Parent Counseling}

\author{
Cecilia Villarreal Montoya ${ }^{1}$ \\ Universidad Nacional \\ División de Educación para el Trabajo \\ Centro de Investigación y Docencia en Educación \\ Heredia, Costa Rica \\ ceciliavillarrealm@gmail.com \\ Ana Lucía Villalobos Cordero ${ }^{2}$ \\ Universidad Nacional \\ División de Educación para el Trabajo \\ Centro de Investigación y Docencia en Educación \\ Heredia, Costa Rica \\ avil@una.cr \\ Ruth Villanueva Barbarán ${ }^{3}$ \\ Universidad Nacional \\ División de Educación para el Trabajo \\ Centro de Investigación y Docencia en Educación \\ Heredia, Costa Rica \\ rvillanu@una.cr
}

Recibido 9 de abril de 2013 • Corregido 22 de agosto de 2013 • Aceptado 11 de setiembre de 2013

1 Orientadora y terapeuta familiar, máster en Estudios de Género, Universidad de Costa Rica. Asesora permanente ad honorem del Proyecto de Extensión Orientando Familias, División de Educación para el Trabajo, CIDE, Universidad Nacional. Lugar de trabajo: Proyecto de Extensión Orientando Familias, División de Educación para el Trabajo, Centro de Investigación y Docencia en Educación, Universidad Nacional, Costa Rica.

2 Licenciada en Orientación, Universidad de Costa Rica. Coordinadora Proyecto de Extensión Orientando Familias, División de Educación para el Trabajo. Académica de la División de Educación para el Trabajo, Centro de Investigación y Docencia en Educación, Universidad Nacional, Costa Rica. Lugar de trabajo: División de Educación para el Trabajo, CIDE, Universidad Nacional.

3 Máster en Psicopedagogía, Universidad Estatal a Distancia. Bachiller y Licenciada en Ciencias de la Educación con énfasis en Orientación, Universidad de Costa Rica. Integrante del Equipo del Proyecto de Extensión Orientando Familias, Académica de la División de Educación para el Trabajo, Universidad Nacional, Costa Rica. Lugar de trabajo: División de Educación para el Trabajo, CIDE, Universidad Nacional. 
Resumen. En este artículo se sistematiza una experiencia de orientación con madres y padres de familia en instituciones educativas de las provincias de San José y Heredia, Costa Rica. La modalidad de "agentes multiplicadores" fue la estrategia utilizada en el proyecto de extensión "Fortalecimiento de la familia" de la División de Educación para el Trabajo (DET) de la Universidad Nacional. Un grupo de profesionales de la orientación y docentes de esta División, capacitó como facilitadoras a un grupo de dieciocho profesionales, tanto de la orientación como de otras disciplinas, en comunicación familiar, en la expresión del enojo, la cosmovisión de las familias, y la planificación de proyectos para trabajar con madres y padres de familia con una metodología participativa, en la cual se tomaron, como insumo, las vivencias de quienes participan en los proyectos de orientación familiar. Los proyectos se pusieron en práctica en nueve instituciones y participaron en total ciento cinco madres y seis padres de familia. En el apartado que resume los logros obtenidos se destaca el impacto del salto metodológico al cambiar "la charla" por la participación de madres y padres con sus propias experiencias vitales en la convivencia familiar. Los aprendizajes obtenidos han sido insumos de utilidad en la ampliación y continuación de este proyecto dirigido a la orientación de figuras progenitoras.

Palabras claves. Orientación familiar, modalidad de agentes multiplicadores, metodología participativa, experiencias de progenitores y progenitoras.

Abstract. This article summarizes a counseling experience with parents from educational institutions in the provinces of San José and Heredia, Costa Rica. The multiplier modality was the strategy used in the Social Action Project entitled "Family Strengthening", from the Education for Work Division (DET) at Universidad Nacional, Costa Rica. A group of counseling professionals and educators from this Division trained eighteen professionals, including counselors and other specialists, as facilitators on topics such as family communication, anger management, family worldview, and project planning in order to work with parents using a participative methodology, which took into account the experiences of participants involved in family counseling projects. Projects were implemented in 9 institutions, and a total of 105 mothers and 6 fathers participated. The section summarizing achievements highlights the impact of the methodological shift from "lectures" to parent participation, where they share their own vital experiences on family life. Knowledge obtained has been essential for expanding and continuing this parent counseling project.

Keywords. Family counseling, multiplier modality, participative methodology, parents' experiences.

Este artículo recoge una experiencia de capacitación con fines multiplicadores que se realizó en el proyecto de extensión: "Fortalecimiento de la familia” (Calderón, Murillo, Villalobos, Villanueva y Villarreal, 2009), de la División de Educación para el Trabajo (DET) del Centro de Investigación y Docencia en Educación (CIDE), de la Universidad Nacional.

Con el propósito de lograr mayor acercamiento a las familias, como ámbito educativo, desde la extensión universitaria, el proyecto "Fortalecimiento de la familia" en un primer momento trabajó directamente con madres y padres de familia y luego, en busca de una mayor proyección e impacto, cambió a la modalidad directa por la de agentes multiplicadores. Esta actividad se denominó "Talleres para facilitadoras", la cual se presenta como una experiencia de "orientación familiar" en cuanto fue un proceso planificado (con teoría y metodología propia 
de esa especialidad disciplinar). Esta facilitación implicó asumir la dirección de un proceso, teniendo claro los objetivos y buscando su cumplimiento. Fue un rol de liderazgo compartido con el grupo, su función principal fue promover el desarrollo de sus miembros. Además, una vez realizado dicho taller, se evaluó tanto la experiencia de capacitación en sí misma, como también la réplica que realizaron las facilitadoras con grupos específicos de madres y padres de familia o personas encargadas de infantes de la provincia de Heredia y San José.

Dicha capacitación se enmarcó en la función formadora que cumple la familia, ya que es precisamente en la cotidianidad familiar donde se adquiere una cosmovisión del mundo relacional $y$, además, se aprenden modelos para asumir tanto la paternidad como la maternidad. La experiencia ha demostrado que la acción formativa de las "familias de origen" (donde nacemos y crecemos), dicta los mandatos y prepara para el cumplimiento de los roles, las funciones y conductas de los hombres y de las mujeres; en otras palabras, allí se dictan y reproducen modelos estereotipados que dan forma a los estilos de crianza, los cuales determinan, en gran medida, la vida intrafamiliar. Por ser las familias espacios educativos, se puede influir en ellas mediante procesos orientadores planificados para el mejoramiento de las prácticas educativas intrafamiliares, con especial énfasis en el mejoramiento de la comunicación y la resolución de conflictos en familia.

\section{Antecedentes}

El proyecto de extensión universitaria "Fortalecimiento de la Familia" inició en el 2002 con la intervención directa a figuras parentales y maternales, por parte de un grupo de académicas y académicos, profesionales en orientación, de la DET, quienes visualizaron el proyecto dentro de la concepción de "Escuela para padres" en las instalaciones del CIDE.

La riqueza de esa experiencia hizo pensar en estrategias metodológicas que permitieran una mayor proyección y cobertura del proyecto. Es así como en el año 2008 se planificó y desarrolló un proceso de capacitación a profesionales de diversas áreas de la educación y afines, con el propósito de que este grupo replicara la experiencia en los centros educativos donde laboraban. En ese mismo año se realizó una invitación abierta a participar en el proyecto tanto a profesionales de instituciones educativas de primaria, como a grupos comunales organizados y también a estudiantes de la Carrera de Orientación de la DET del CIDE de la Universidad Nacional.

La iniciativa fue acogida por:

- Cinco escuelas de la provincia de Heredia: Escuela José Ramón Hernández, Escuela José Figueres Ferrer, Centro Educativo Villalobos, Centro de Enseñanza Especial de Heredia y Escuela Braulio Morales.

- Una escuela de la provincia de San José: Escuela Las Brisas del Virilla. 
URL: http://www.una.ac.cr/educare

CORREO: educare@una.cr

- Una organización religiosa: La Iglesia Pueblo de Dios.

- La Asesoría de Educación Especial y Desarrollo Educativo de la Dirección Regional de Heredia del Ministerio de Educación Pública.

- La Biblioteca Infantil "Miriam Álvarez" (conocida también como “El trencito").

Durante el primer año se realizó la capacitación directa con las facilitadoras de las instituciones ya mencionadas y, en el segundo año, se les brindó asesoría y compañía metodológica para el desarrollo del proyecto que planificaron para madres y padres de familia como producto de la capacitación.

\section{Principios teórico-metodológicos que guiaron la experiencia práctica}

\section{Las familias como instancias formadoras}

La experiencia realizada se posicionó en algunos fundamentos sistémicos sobre las familias. Predomina el modelo de validación humana de Satir (2005), quien destaca la responsabilidad de la familia en la formación de las generaciones al compararla con una fábrica, cuyo producto son las personas.

En el planteamiento sistémico, las familias son caracterizadas fundamentalmente por la diversidad relacional, o sea, cada familia en su historia generacional va construyendo estilos y patrones de interrelación que le proporcionan la propia identidad. Entonces, con este enfoque, no es posible definir un perfil "normal" de las familias, aunque sí se plantean tareas que esta institución social debe cumplir, tales como el cuido de las personas y el aprendizaje de diversas formas para lidiar con los conflictos, mediante formas adecuadas para la comunicación que favorezcan el desarrollo de la familia como un todo (Beavers y Hampson, 1995); sin embargo, cada familia lo asume de forma peculiar. En este mismo sentido, Richardson (1993) refuerza la premisa de que todas las familias son iguales al mismo tiempo que cada una es diferente de las otras. Lo que se quiere decir es que, a pesar de que en apariencia las familias presentan una configuración y un desarrollo similar, cada una se distingue por la forma y el estilo en que cumple las tareas asignadas por la sociedad.

Según la tipología de los sistemas, y retomando a Satir (2005), la familia como sistema abierto es flexible a los cambios en los momentos que el desarrollo de la familia lo requiera. Además, sus integrantes hacen uso de la comunicación directa, con lo cual se un ambiente agradable para la convivencia interna y buena relación con otros sistemas de la sociedad. Por el contrario, la familia cerrada se caracteriza por tener un manejo dificultoso para el cambio y la comunicación. Al mismo tiempo, la forma de comunicarse de estas familias es bastante confusa e incongruente y mantienen reglas de relación muy rígidas que dificultan la interacción entre sus integrantes y la del sistema familiar con otros sistemas. 
Lo ideal para la misma autora es que las familias practiquen la comunicación niveladora o adecuada. Esta permite a las personas expresar sus ideas y sentimientos con claridad y en forma directa. Sin una comunicación adecuada, las familias se convierten en espacios nocivos para el crecimiento humano, lo cual es especialmente preocupante, porque es en las familias donde las personas aprenden las principales habilidades para la vida.

Las habilidades para la convivencia se reflejan en las formas de actuar de las personas y, en ese sentido, es que Watzlawick, Beavin y De Avila (2002) le dan la condición de conducta a toda comunicación (sea esta verbal o no verbal). Al concebirse la comunicación como conducta, los modelos inadecuados de comunicación aprendidos pueden cambiarse por formas adecuadas. Dicha conversión puede realizarse al observar e identificar los propios modelos inadecuados de comunicación que se utilizan y, en segundo lugar, reconociendo la existencia de alternativas comunicacionales humanizantes y facilitadoras del desarrollo integrado de las personas.

Tanto Sr. Dinkmeyer, Mckay y Jr. Dinkmeyer (1998), como Watzlawick et al. (2002), plantean que la comunicación no se limita al intercambio de información, sino que en cada intercambio de contenido se expresan sentimientos. Este tipo de comunicación afectiva requiere de una escucha reflexiva, la cual se basa en el respeto, e implica esfuerzo por reconocer los sentimientos que están detrás de las palabras expresadas y procurar entender lo que se está tratando de decir. Además, es importante, para las personas comunicantes, poner atención y, sobre todo, disponerse a la comprensión de los mensajes verbales y no verbales.

Otro postulado teórico relevante que enmarca el desarrollo de este proyecto de extensión universitaria hace referencia al hecho de que las familias no están aisladas: todas ellas son parte del mesosistema de la sociedad (López y Escudero, 2003). Esto quiere decir que las familias se interrelacionan entre sí y con las instituciones educativas, los espacios laborales, las iglesias y los grupos comunitarios (entre otros). En la dimensión mesosistémica, las universidades públicas juegan un papel preponderante en cuanto al fortalecimiento de la alianza entre las familias y los centros educativos, por medio de esfuerzos concretos respecto a la educación y la orientación de las familias.

\section{La modalidad grupal como estrategia de intervención con grupos de madres y padres de familia}

La modalidad de intervención grupal para padres y madres de familia permite el aprendizaje colectivo a partir de las experiencias individuales, en la medida en que estas sean compartidas y retroalimentadas entre sus participantes. Esta forma de intervención contempla aspectos de todo el grupo, quienes participan "... tienen necesidades, viven problemas afines o semejantes" (García, 2001, p. 341), que se originan en el momento evolutivo de vida y las tareas que este demanda. 
URL: http://www.una.ac.cr/educare

CORREO: educare@una.cr

Por ello, en conjunto, madres y padres de familia podrán encontrar formas más adecuadas para atender las situaciones que comparten en la crianza de la prole. Participar de la experiencia grupal, como lo menciona Sanz (2007), permite "la oportunidad de aprender una serie de competencias como escuchar de forma empática, pensamiento crítico, expresión de sentimientos, solución de problemas y sentido de la responsabilidad y habilidades de autogestión" (pp. 114-115), estas mismas pueden ser aplicadas durante las sesiones y "transferir[se], después, a la vida fuera del grupo" (p. 115).

Esta modalidad de intervención orientadora implica llevar a cabo un proceso sistemático y coherente entre sus partes. En este sentido Sanz (2007), haciendo referencia a Blum, anota que el contexto grupal promueve la autoestima y estimula la consecución de metas de fácil alcance, con el fin de lograr una mejora constante de quienes participan.

Es conveniente que la guía y conducción del proceso la brinde una persona capacitada en cuanto a la temática específica que reúne al grupo, y con un buen manejo de grupo, según un enfoque o modelo específico de intervención.

El rol de quien conduce los procesos grupales es de gran importancia, como su nombre lo sugiere, es la persona que facilita la interacción y el aprendizaje entre las personas participantes estimulando su participación. Además, observa y atiende las situaciones conflictivas del grupo, organiza las actividades, aporta conocimiento en busca de la promoción de cambios que favorezcan el crecimiento individual y grupal, pero no se impone. Nunca se debe manipular la dinámica del grupo; por el contrario, se trabaja con ella: esto implica que el grupo, con el apoyo de quien facilita, pueda entender la forma en que interactúa entre sí en términos de patrones relacionales. De esta manera se puede trascender en el rompimiento de patrones inadecuados y en grupo apoyarse abiertamente en la manutención de formas adecuadas para una convivencia más satisfactoria.

El trabajo con grupos es una herramienta muy útil en la educación con personas adultas, en la medida que permite el incremento del pensamiento, la autogestión, la reflexión y la exploración de los estilos de crianza, en este caso, además del mejoramiento de la calidad de vida de las personas que integran el grupo.

Es necesario tener presente que las personas adultas dentro de los grupos tienen intereses definidos y diversos, situación que se puede tomar como un reto por quien facilita en busca de la satisfacción, total o parcial de las metas individuales-grupales. Entonces, en la medida en que el aprendizaje sea satisfactorio y significativo en su realidad relacional (de la persona consigo misma y con otras personas), crecerá el interés en la profundización de las propias experiencias de vida.

De acuerdo con Sanz (2007), participar en un grupo de orientación tiene beneficios. Entre ellos se pueden mencionar los siguientes: expresar situaciones que preocupan, aprender a confiar, trabajar conjuntamente, brindar apoyo mutuo, tomar decisiones y lograr un mayor conocimiento de sí mismas. 
Todos esos beneficios justifican la necesidad de crear espacios donde los padres y las madres puedan reunirse para conversar y trabajar aspectos relacionados a la cotidianidad familiar y, en especial, al ejercicio de la parentalidad. En estos grupos, quienes participan aprenden unos de otros, se apoyan, estimulan y favorecen las condiciones para el compromiso en la toma de decisiones; además, en el caso de personas tímidas, reduce la tensión de singularizar su situación.

Las personas que asumen el rol facilitador deben prepararse con anticipación para asumir la tarea de conducción de un grupo. Fuhrmann y Chadwick (1998) recomiendan tomar en cuenta los siguientes aspectos para que la labor facilitadora sea más efectiva:

1. Habilidad para entender diferentes formas de pensamiento y abrirse a nuevas perspectivas de vida.

2. Habilidad para no enjuiciar y aceptar las diferencias entre las personas.

3. Equilibrar las distancias afectivas entre quienes conforman el grupo, de modo que todas las personas sientan libertad para acercarse a quien facilita el trabajo del grupo.

4. Habilidad para liderar el proceso de modo que cree un contexto para el cambio.

5. Habilidad para perfeccionar herramientas que le permitan dar instrucciones claras.

6. Habilidad para autoevaluar su desempeño y aprender de la experiencia.

El rol facilitador puede resumirse en el compromiso de "renunciar a una serie de roles como autoridad, consejero y solucionador de problemas y, por el contrario, convertirse en profesionales que estimulen, motiven y enseñen habilidades, realicen intervenciones eficaces y confíen en la posibilidad de que los miembros del grupo se ayuden unos a otros" (Sanz, 2007, p. 113).

La cooperación y el aprender de las vivencias de otras personas permiten a madres y a padres de familia la apertura al abanico de posibilidades, y a la diversidad de soluciones o salidas de situaciones comunes, en el ejercicio del liderazgo para cumplir con la tarea de disciplinar a la descendencia.

\section{Estrategia metodológica utilizada en la capacitación al grupo de facilitadoras}

En la capacitación brindada participó un grupo de 18 mujeres profesionales, provenientes de diversos campos, entre ellos: educación, trabajo social, psicología, derecho, administración educativa, secretariado, bibliotecología y orientación. Además participaron amas de casa y estudiantes de la Carrera de Orientación de la Universidad Nacional. 
URL: http://www.una.ac.cr/educare

CORREO: educare@una.cr

Se aplicó una metodología vivencial y participativa, de tal manera que el proceso les permitió, en primer lugar, la comprensión de principios teóricos sobre familia y de intervención educativa con estas. En segundo lugar, las participantes tuvieron la oportunidad de poner en prácticas los nuevos conocimientos primero en sus propias familias, tal y como ellas mismas lo compartieron al expresar los logros generados de los cambios que decidieron realizar, y luego como facilitadoras al servicio de las "Escuelas para padres y madres" en el propio ámbito laboral.

El grupo de participantes tuvo la oportunidad de vivenciar, primeramente, el proceso de orientación familiar construido por quienes las capacitaron, para luego, multiplicar esa experiencia.

\section{Proceso de capacitación al grupo de facilitadoras}

Con el propósito de organizar la capacitación, por parte del grupo de profesionales de orientación de la DET encargado de dicha tarea, se visualizaron dos etapas bien definidas: una primera fase de formación teórico-práctica y una segunda fase de asesoría en la réplica de la experiencia de capacitación con un grupo de madres y padres de familia en sus centros de trabajo.

\section{Etapa: Revisión teórico-metodológica para la intervención con familias}

Intercalando los principios teóricos con las propias vivencias cotidianas de las participantes, se trabajaron las siguientes temáticas:

1. La cosmovisión sobre familia: mediante el análisis del significado de ser madre o padre, las participantes se dieron la oportunidad de explorar el significado y el rol maternal que cumplen en sus propias familias, al mismo tiempo que se revisaban los contenidos teóricos respectivos. Se analizó también la importancia del rol paterno y la necesidad de que los varones lo asuman y, por ello, fue necesario analizar las demandas de la cultura patriarcal expresada en estereotipos, comportamientos y formas de reaccionar según la condición de género en la asunción de los roles y las responsabilidades propias de la convivencia familiar.

2. La comunicación y la expresión del enojo: se revisaron modelos de comunicación inadecuados en las relaciones familiares y las formas de asumir el manejo del enojo en las familias. Se enfatizó en el papel de la comunicación afectiva como pilar importante para establecer relaciones saludables en la familia.

3. La planificación de proyectos para madres y padres de familia y el rol facilitador de esos procesos educativos: las participantes fueron construyendo un plan para desarrollar 
en la propia institución donde laboraban, al mismo tiempo que se fueron clarificando las habilidades facilitadoras para trabajar con grupos de figuras parentales. Se enfatizó en la conducción de grupo, en el papel de quien la asume y en las tareas propias para facilitar un grupo de esta naturaleza. Se retomaron las habilidades para la intervención a familias, lo anterior debido, por un lado, al compromiso que significa liderar procesos familiares $y$, por otro lado, por la diversidad de formación disciplinar del grupo de facilitadoras.

\section{Etapa: intervención educativa con madres y padres de familia}

En esta fase las participantes en la capacitación asumieron el rol como facilitadoras durante la implementación del plan de trabajo previamente planificado. El equipo capacitador les brindó acompañamiento en el desarrollo de los planes mediante la ampliación de temas y la discusión de los avances y dificultades enfrentadas. Con este propósito, el grupo de facilitadoras se reunieron cada dos semanas con el equipo capacitador.

La evaluación se realizó durante el proceso de capacitación, al finalizar cada una de las sesiones cada participante tuvo la oportunidad de externar su opinión respecto a la relevancia del tema abarcado, y de los aprendizajes personales y profesionales obtenidos. Además, en dos oportunidades se realizó la valoración del proceso completo (estas se realizaron en forma oral).

Durante esta segunda etapa del proceso de capacitación, el equipo capacitador realizó visitas a las instituciones participantes donde se entrevistaron con los directores o las directoras y con las facilitadoras. Finalmente, las facilitadoras escribieron el informe que presentaron en forma oral y escrita en una sesión de cierre donde se discutieron los logros obtenidos y las dificultades enfrentadas.

\section{Logros y dificultades relevantes del proceso de capacitación a las facilitadoras}

Para organizar los resultados alcanzados en este proceso de capacitación se respetó el orden de cada una de las etapas del proceso descrito anteriormente.

\section{Etapa: Revisión teórico-metodológica para el trabajo con familias}

Durante esta etapa, las facilitadoras emprendieron el aprendizaje de una estrategia para facilitar procesos de orientación con madres y padres de familia. Esta modalidad grupal, que se centró en la participación de las figuras progenitoras con sus experiencias de vida en la convivencia familiar y no en impartir charlas o conferencias, retoma tanto el aprendizaje de contenidos, como también la expresión de las emociones, sentimientos, ideas y cambios significativos personales y en sus propias familias. Todos esos elementos juntos hicieron posibles avances significativos en las participantes una vez que asumieron el rol facilitador tanto en el ámbito profesional como laboral. 
URL: http://www.una.ac.cr/educare

CORREO: educare@una.cr

En sus propias palabras, las facilitadoras expresaron:

"Yo personalmente me siento muy motivada, esta capacitación nos abrió paso para revisar y reforzar cosas en lo personal para llevar este trabajo a las familias de mi institución que también mejoraron significativamente".

"Yo de todas mis compañeras he aprendido a pesar de mi profesión (psicóloga). Uno se enriquece, qué bonito, como piensan, lo que dice las compañeras que están en esta capacitación".

En relación con la oportunidad de renovar conocimientos, otras participantes mencionan:

"Es un espacio de refrescamiento porque uno al estar tan metido en la institución trabajando con la misma población tiende a caer en la pasividad yno se refresca en nuevos conocimientos".

"Siento que al compartir nuevas ideas, se le encienden a uno chispitas para llevarlas al ámbito laboral, logrando cambios importantes y novedosos".

"Creo que la metodología de las sesiones pueden brindar más tiempo a la expresión de sentimientos y profundizar en cada persona y en su familia".

"Me llevo conocimientos que calaron a nivel personal y además tengo la oportunidad de ponerlos en práctica y compartir con familias de mi propia institución, es todo un reto profesional y personal".

En forma compartida, las facilitadoras concuerdan en que el ambiente generado en las sesiones (debido a la metodología utilizada) facilitó la creación de un espacio de confianza para el aprendizaje de nuevos temas, se brindó apertura a la expresión de sentimientos y de preocupaciones, pero sobre todo una gran motivación y disposición de poner en práctica lo aprendido.

Durante las sesiones en que se abarcaron temas relacionados con la cosmovisión de la familia y los roles materno y paterno en ella, las participantes especificaron que la forma en que se trabajó les permitió reconceptualizar la visión de familia, además de ampliar la comprensión y explicación de la propia experiencia de la vida en la familia. Ellas afirmaron:

"He aprendido que todas las familias son diferentes, no todas tienen las mismas dificultades o situaciones, que no soy solo yo la que está padeciendo... También veo que hay estrategias para tratar situaciones sin llegar al castigo o a situaciones frustrantes". 
URL: http://www.una.ac.cr/educare CORREO: educare@una.cr

"El taller me permitió compartir situaciones familiares vividas como madre e implementar cambios al respecto para el beneficio de la familia".

"Este espacio a mí me encantó porque aquí puedo ser mamá, yo no tengo ese espacio fuera de acá para compartir al respecto. Aquí puedo expresarme sin miedo".

En la evaluación oral de estas sesiones, las participantes plantearon que pudieron identificar el rol materno en sus propias familias $y$, además, se cuestionaron la relación existente entre el rol que juegan en la familia y el impacto de este en el rol profesional. Situación que les permitió humanizar el rol profesional al comprender que la razón y la emoción son inseparables y que el aprendizaje se obtiene en convivencia.

\section{En cuanto al tema relacionado con la comunicación y el manejo del enojo}

Las participantes tuvieron la oportunidad de explorar su propia convivencia familiar en cuanto a la comunicación y el manejo de emociones como el enojo. En forma simultánea, las facilitadoras adquirieron el aprendizaje de estrategias para trabajar la comunicación y el enojo con madres y padres.

Ellas opinan:

"La capacitación me permitió confrontar situaciones familiares vividas e implementar cambios a nivel familiar".

"Me ha ayudado a no enojarme tanto....cada vez que yo venía aquí decía esto es lo que estoy haciendo, yo ahora soy más fresca, me enojo menos porque estaba cometiendo muchos errores".

"Ahora puedo valorar a mi esposo y con mi hija soy más tolerante porque yo era muy exigente".

"El compartir los materiales con familiares, también ha influido en los cambios obtenidos en sus familias". Y que además, "fueron cambios que funcionaron para lograr mejoraras familiares".

Con esta claridad y grado de sensibilización, las facilitadoras fueron visualizando nuevas opciones para la labor con madres y padres de sus instituciones.

\section{Respecto a la formación como facilitadoras}

Las participantes concordaron en que el modelaje de quienes facilitaron las sesiones del proceso de capacitación permitió una mayor comprensión de la actitud facilitadora, la forma de 
URL: http://www.una.ac.cr/educare

CORREO: educare@una.cr

dar las instrucciones, el uso de los recursos y herramientas disponibles y la disposición constante a valorar las acciones que se realizaron en los diversos momentos del proceso de capacitación.

Las participantes afirman que las sesiones les ofrecieron "un refrescamiento con nuevos temas para trabajar con madres y padres". Además de "la posibilidad de examinar el trabajo que habíamos venido realizando" y a "valorar cuáles son las fallas y debilidades al compararlo con la capacitación recibida", así como también valorar que "hemos podido aprender nueva teoría para el trabajo con madres y padres de familia". También reconocieron "el aprendizaje de nuevas formas y estrategias para trabajar temáticas ya conocidas", lo que ha ayudado a fortalecer las escuelas de padres y madres que lideran.

\section{En lo referente a la planificación de los proyectos institucionales}

Con la guía del equipo académico del proyecto "Fortalecimiento de la familia", las facilitadoras concretaron nueve proyectos, uno por institución, para trabajar con madres y padres de familia.

En general, los proyectos replicaron el proceso de capacitación vivenciado, estos iniciaron con la revisión de la concepción de "familia" de las personas participantes. Se tomó muy en cuenta la diversidad de las familias, el cumplimiento de los roles materno y paterno, las condiciones y los recursos de cada familia en su propio contexto.

Luego retomaron el tema de la comunicación intrafamiliar, el manejo del enojo, y los problemas comunicacionales en las relaciones entre quienes conforman las familias; enfatizaron en la comunicación afectiva y en la convivencia intrafamiliar.

Adicional del proceso expuesto, la Escuela Centro Educativo Villalobos de Heredia, desarrolló el tema de la sexualidad en la familia.

Una de las asesoras de educación especial de la Dirección Regional de Heredia planteó y desarrolló un curso con profesionales de esa disciplina utilizando el paradigma de la inclusión.

\section{Etapa: intervención con madres y padres de familia}

Durante esta etapa se pusieron en práctica los nueve proyectos planificados en las instituciones ya mencionadas. Las facilitadoras organizaron en cada centro de trabajo un grupo de madres y padres de familia quienes participaron en el proyecto; las instituciones que ya contaban con "Escuela para padres y madres" incluyeron en el nuevo proyecto a esta. Otra modalidad fue coordinar con el EBAIS de la localidad como una forma de trabajar en equipo, aprovechando las redes sociales existentes.

Los proyectos tuvieron buena acogida por parte de la dirección de las instituciones, así como por la población meta; participaron en total ciento cinco (105) madres y seis (6) padres de familia. Es importante destacar que la participación fue mayormente femenina, los padres 
que se unieron a sus esposas pertenecen a la Iglesia Comunidad Cristiana Pueblo de Dios (5) y a la Biblioteca infantil Miriam Álvarez Brenes "Trencito infantil"(1).

Por condición socioeconómica, las madres y los padres que participaron se ubican en los sectores medios y bajos, dos de las comunidades participantes son definidas como urbano marginales. Además, respecto a la edad, este grupo se encuentra entre los 19 y los 54 años, con un promedio de 4 hijos por familia.

Las personas que participaron son oriundas de la provincia de Heredia y de San José (Brisas del Virilla). Se desplazaron desde las comunidades San Rafael, San Joaquín, Bajos de los Molinos, Jardines, Mercedes Norte, Santo Domingo, San Pablo, San Isidro, Lagunilla, Santa Rosa, Guararí, urbanización Lilliana, Precario Palacios, La Radial Sauces y del Residencial Verona.

Además, en cuanto al nivel educativo, la mayoría de madres y padres completaron la primaria; se destaca un número reducido de mujeres con formación universitaria.

Respecto al estado civil, la condición de matrimonio y la de unión libre predominan; aunque también participaron mujeres separadas, divorciadas y solteras jefas de hogar. En su mayoría, las mujeres que participaron atienden los oficios del hogar y el cuido de las hijas e hijos.

\section{La puesta en marcha del proyecto en las instituciones participantes}

En cada una de las instituciones se realizó la invitación abierta a madres y padres de familia de la comunidad educativa o religiosa, según fuera la población meta. Se formaron grupos pequeños (entre 5 y 15 personas) y se trabajó en horarios diurnos, debido a que fueron mujeres, en su mayoría, las que mostraron interés en participar.

Cada institución realizó un promedio de cinco sesiones con madres y padres, las cuales siguieron, en su mayoría, las temáticas y el formato de la capacitación recibida de las facilitadoras; otras innovaron con temas que, aunque no habían sido trabajados directamente en las capacitaciones, los consideraron necesarios para la población atendida.

Estas son algunas de las preocupaciones que las facilitadoras experimentaron al inicio del desarrollo de los proyectos:

- Temor de aplicar una nueva estrategia metodológica que implicaba ayuda mutua de las personas integrantes del grupo. Es decir, al facilitar el grupo no de forma directiva sino participativa les parecía que perderían el control al permitir que la dinámica del grupo asumiera un rol protagonista.

- Incertidumbre por la conformación del grupo, en especial en aquellas instituciones en que el apoyo de la administración fue un poco débil. Lo plantearon así: "Este año tuve mucho miedo de empezar con los padres de familia debido a que no tenía apoyo institucional y que eran pocas personas las que se habían inscrito", "topé con frustración inicial porque no llegó la cantidad de padres y madres esperada". 
URL: http://www.una.ac.cr/educare

CORREO: educare@una.cr

- Temor de que atendieran el llamado las familias menos necesitadas, les preocupaba el hecho de que asistieran las madres que desde su perspectiva, "tenían relaciones más sanas dentro de la familia".

Una vez iniciado el proceso, las mismas facilitadoras se sorprendieron de la facilidad con que brotó la habilidad empática de ellas con el grupo y entre las participantes entre sí; en gran parte el mostrar vulnerabilidad y enfrentar el mito de la perfección en las familias.

La mayoría de las facilitadoras también coincidieron en que, cuando actuaron como tales: "Se promovió la oportunidad de que las señoras no solo se percibieran como madres sino también como personas con necesidad de socializar con otras adultas y de satisfacer necesidades de recreación y actividad física".

Según las facilitadoras, con el desarrollo del proyecto, el logro mayor fue la participación y la constancia en cuanto a la permanencia de las madres y los padres durante su desarrollo en las instituciones.

En segundo lugar, las facilitadoras consideran que la metodología participativa utilizada, permitió mantener un ambiente de confianza y de apertura a la expresión de sentimientos, y de preocupaciones genuinas de quienes compartieron el proceso.

En tercer lugar, según el reporte de las facilitadoras, fue increíble la disposición de las madres a poner en práctica, en sus familias, lo aprendido durante las sesiones. Ellas mismas fueron planteando los cambios que se iban dando en sí mismas y en el grupo familiar. Con gran facilidad fueron expuestas las dificultades en la convivencia familiar y la forma en que fueron logrando mejorar las relaciones al esforzarse en la puesta en práctica de la comunicación efectiva y las estrategias para un mejor manejo del enojo. También especificaron que el aprender a escuchar antes de actuar les ha permitido aconsejar con mayor sabiduría a la prole.

Además, la reflexión sobre el rol materno y paterno permitió a las participantes compartir sus experiencias como responsables de la crianza, en especial para las mujeres solteras o divorciadas. Ellas se encuentran muy aisladas cumpliendo con la función maternal-crianza. Se resiente la compañía de los varones, quienes no asumen la función paternal-crianza. Sin embargo, ante esta realidad, una señora especificó haber logrado "estabilidad en mi familia. Tengo clara mi posición en la familia. Conocer una forma de compartir... las experiencias en una familia ... La experiencia me inspira un plan de vida".

También, la posibilidad de las madres de ponerse en el lugar de sus hijos e hijas, que brindaron algunos de los ejercicios realizados, impactó a estas figuras progenitoras, pues les permitió mayor sensibilidad para la educación de la prole, en especial, con adolescentes. Según las facilitadoras, las madres "tuvieron la oportunidad de ponerse en el lugar de sus hijos e hijas y tener otra perspectiva para la educación de la prole". 
Según las facilitadoras, las sesiones de trabajo con las madres se convirtieron en un espacio en donde ellas expresaron frustraciones y preocupaciones que viven como madres, con poco apoyo de los maridos y algunas con serios problemas económicos. Algunas de ellas buscaron atención individualizada, aunque el proyecto no contemplaba esta modalidad de intervención.

Finalmente, las madres participantes, estimuladas con la posibilidad de "corregir sin pegar", solicitaron a las instituciones la continuación con proyectos de formación en educación a la familia, dando especial énfasis en la disciplina para la crianza. Esta petición ha sido avalada por el personal de supervisión y por otras personas funcionarias de las instituciones, quienes consideran que mediante este tipo de proyecto se amplía la relación centro educativo-familia.

Para las facilitadoras, este tipo de intervención novedosa les permitió alcanzar mayor "crecimiento personal y profesional", además de haber podido "integrar a las madres interesadas al ámbito escolar". Destacan que les fue de gran utilidad haber aplicado el "conocimiento adquirido para el fortalecimiento en la relación con mi propia familia". Para ellas, "el haber recibido un conocimiento amplio" les permitió también "ayudar a los padres de familia de mis alumnos y alumnas en cuanto a la detección de violencia intrafamiliar, problemas de conducta, problemas de aprendizaje, manejo de diversas situaciones, entre otros." Todas estas, situaciones cotidianas en las familias que acuden a esos centros educativos.

La mayoría de las facilitadoras concuerdan en que el crecimiento personal y profesional es un logro del proyecto ejecutado. Y lo expresan de la siguiente manera: "Obtuvimos conocimientos que calaron a nivel personal". "Verifiqué que es posible en nuestro centro educativo desarrollar talleres de varias sesiones". "Cuento con material muy valioso y es posible volver a impartir los talleres con diferentes padres de familia".

Por otra parte, las participante pusieron también en práctica lo aprendido en la capacitación recibida en el trabajo de aula y en otras funciones laborales; y puntualizan las estrategias para mejorar la comunicación de sentimientos, controlar el carácter, no culpabilizarse, tolerar otros puntos de vista, dar ideas sin imponerlas, tener conciencia de que nadie es perfecto $y$, sobre todo, el saber escuchar.

El taller para facilitadoras produjo, además, en la mayoría de las participantes, sentimientos positivos como satisfacción, felicidad, entusiasmo, entre otras; aunque algunas de ellas mencionan también el haber experimentado ansiedad al no saber cómo iba a concluir el proceso e impotencia por sentirse poco apoyadas por parte de la administración del centro educativo. Con respecto a los sentimientos generados en ellas durante el proceso, dos participantes mencionan:

"Me ha encantado participar de este largo proceso, considero que es importante y necesario llegar a los padres por medio de este tipo de talleres".

"Cansancio, porque requiere entrega de nuestra parte. Un poco de satisfacción de ver que el esfuerzo valió la pena. Felicidad porque me permitió acercarme exitosamente a la comunidad". 
URL: http://www.una.ac.cr/educare

CORREO: educare@una.cr

Para realizar este tipo de actividades extra clase, las profesionales debieron disponer de tiempo que les significó recargo de sus labores y búsqueda de los materiales adecuados, la dedicación de ellas fue un gran aporte para el mejoramiento de las familias, que se vio reflejado, según las profesionales, en el desempeño escolar de sus estudiantes.

\section{Acompañamiento y seguimiento a las facilitadoras por parte del equipo capacitador}

El equipo capacitador brindó acompañamiento a las facilitadoras en el proceso de ejecución de los proyectos. Este se realizó mediante:

- Sesiones presenciales, cuatro horas cada dos semanas en las instalaciones del CIDE, en estas se monitoreó el avance, logros, dificultades y la diversidad de formas en que se facilitó el progreso en cada institución.

- Comunicación constante a través de los medios electrónicos.

- Visitas de seguimiento a las instituciones por parte del equipo capacitador.

Según este equipo, las sesiones grupales realizadas fueron de especial provecho, pues permitieron a las participantes "la posibilidad de escuchar a las compañeras, el aporte de diversas ideas y perspectivas desde el quehacer y formación de cada una. Situación que ayudó a fortalecer y a enfrentar con ánimo algunas circunstancias que se presentaban. También se considera que fue un espacio de refrescamiento personal. Se destaca el trabajo enfocado de manera más humana".

Los medios tecnológicos fueron también un apoyo en la etapa de seguimiento, especialmente el correo electrónico para "cuando tenía alguna duda la enviaba por correo, rápido tenía la respuesta, siempre hubo ese contacto".

En las entrevistas realizadas a las instituciones, las directoras y los directores expresaron su excelente apreciación por la puesta en marcha del proyecto y agradecieron el hecho de que se les tomara en cuenta en este tipo de capacitaciones.

Para finalizar el proyecto, el grupo facilitador realizó un informe final (por institución) donde se recogió la experiencia en términos de logros, dificultades y limitaciones de las diferentes etapas del proyecto. Este fue expuesto en forma pública, con la presencia de autoridades del CIDE y de las instituciones participantes.

\section{Sugerencias de las facilitadoras para mejorar la capacitación}

Por diversos medios y en diferentes momentos del proceso de capacitación, las facilitadoras plantearon los siguientes aspectos para mejorar: 
Poner reglas claras de participación para optimizar el uso del tiempo evitando las participaciones o comentarios extensos. Dicha sugerencia aplica tanto para las actividades realizadas en la etapa de capacitación como para la etapa de ejecución en que ellas participaron como facilitadoras.

Un pequeño grupo, sugirió implementar en algunas sesiones actividades más dinámicas y creativas. Cabe anotar acá que lo relevante es que el ejercicio facilite el logro de la meta a alcanzar, no se trata de realizar el ejercicio por sí mismo, sino que por sus alcances respecto a los propósitos propuestos.

Respecto al interés por otros temas, el grupo de facilitadoras sugirió la necesidad de profundizar sobre: trabajo en equipo, trabajo con adolescentes y conocer más técnicas de intervención orientadora.

Una participante recomendó que para optimizar el logro en las sesiones se realizaran menos actividades para dar mayor tiempo a la expresión de sentimientos. Sobre este aspecto menciona: "Sé que es difícil pero cuando se remueven sentimientos y se cortan no es lo más recomendable... dejar más tiempo... tal vez no recargar mucho las sesiones". Una petición parecida hacen varias madres participantes de una de las instituciones, quienes plantearon la necesidad de tratar con mayor profundidad cada uno de los temas tratados. Este es un aspecto muy importante y digno de ser tomado en cuenta, ya que las familias cuentan con pocos espacios para reflexionar sobre la propia convivencia.

Otro aspecto importante, según las facilitadoras, es ofrecer actividades simultáneas para las niñas y los niños que las madres llevan a las sesiones por no tener con quien dejarlos en el hogar.

Queda por resolver la integración de los padres de familia a este tipo de programa. Ellos muestran menor anuencia e interés a participar. Según las madres, ellos descargan la responsabilidad de la crianza en ellas.

Es conveniente considerar, dentro del proyecto, la orientación individual a las madres que lo requieran o bien que así lo soliciten. Aunque de alguna forma esto se realizó, es necesario planificar esos espacios.

Las docentes solicitaron, al finalizar el proyecto, introducir en el proceso de capacitación lo referente a "la intervención en crisis" debido a que se les presentaron situaciones de este tipo.

\section{Consideraciones finales}

Finalizada la experiencia, es importante plantearse, para la reflexión, tres ideas fundamentales para favorecer la intervención grupal con madres y padres de familia: 
URL: http://www.una.ac.cr/educare

CORREO: educare@una.cr

La primera idea "Aprendiz-facilitar". Para favorecer un proceso dentro de un marco de orientación de procesos en las familias, es necesario tener la modestia de sentirse aprendiz, es necesario tener la claridad de que siempre estamos aprendiendo y lo hacemos mediante el análisis y reflexión de las experiencias cotidianas con la intención de buscar y encontrar mejores formas de relación. La gran ventaja de trabajar en grupo con madres y padres de familia con enfoque vivencial es la riqueza de compartir la diversidad de situaciones que se enfrentan día con día. En este sentido, se mantiene mucho interés y aportes creativos en busca de mejores formas de educar y educarse para cumplir con el rol de formadoras y formadores en el ámbito familiar. Las facilitadoras fueron las primeras sorprendidas respecto a la participación en las representaciones de papeles y el resto de las actividades que ellas propusieron. El paso de la conferencia a la representación de las dificultades en concreto les fue muy útil y, además, mantuvieron el interés y la participación en las sesiones. Además, en las familias fueron capaces y creativas para facilitar los cambios.

Cada quien asumió la responsabilidad de ser persona y ese camino les llevó, necesariamente, a cuestionar lo aprendido para mantenerlo, deconstruirlo y reconstruirlo. Fue quedando claro el hecho de que cada familia de procreación hereda patrones relacionales de las familias de origen y estas de sus antepasados, y así se logró la comprensión de la forma en que se consagra el famoso "deber ser", aunque no se entiendan los porqués ni los para qué.

El darse la oportunidad de realizar el ejercicio para comprender las formas en que se construyen y se mantienen las relaciones con sus propios familiares, les permitió la pericia a las facilitadoras para guiar el mismo ejercicio con madres y padres de familia.

Por supuesto que esta conclusión se sostiene al integrar la teoría relacional y la metodología que plantea que no se puede enseñar lo que no se sabe hacer. Esta es la mayor enseñanza adquirida por las facilitadoras en esta capacitación. Ellas se centraron en la revisión de su propia familia; guiadas por el equipo capacitador, se contactaron con su propia realidad, mejoraron la convivencia en su propia familia para luego poner ese conocimiento al servicio de otras personas adultas que, en forma similar, fueron descubriendo sus orígenes relacionales y dando espacio al cambio con el uso de teoría sobre comunicación, fundamentalmente.

El segundo tópico clave fue "cambiar-facilitar". Para ampliar la formación respecto a la convivencia en familia, se requiere tener aprendizajes significativos respecto a la forma de leer la familia con nuevos anteojos, además de tener apertura para la exploración y la adquisición de nuevas estrategias metodológicas que lleven al cambio. Estas, necesariamente focalizadas en las interrelaciones comunicacionales. No se trata de decir lo que se debería hacer, sino de realizar o bien visualizar con claridad los patrones 
que deben ser desechados durante la sesión de trabajo grupal. Son acciones poco intelectualizadas, actuadas de tal forma que en una dimensión concreta se experimentan diversas posibilidades para sustituir la comunicación inadecuada. Las facilitadoras mostraron que el haber logrado cambios en la propia familia les proporcionó mayor confianza para facilitar procesos de cambio en los grupos de madres y padres de familia. Para lograr cambios hay que arriesgarse a practicar acciones diferentes a las que hasta ahora se hayan puesto en práctica.

Para la manutención de los cambios se requiere un método para lograrlo. La fenomenología rescata la atención y participación consciente de quienes buscan las transformaciones. Primero, se requiere la comprensión del fenómeno, para luego buscar las sustituciones que garantizarán las transformaciones. Una condición necesaria fue la que lograron las facilitadoras y, luego, madres y padres de familia: permitirse entrar en contacto con su propia situación comunicacional como personas y con sus familias. El que las personas estén en contacto consigo mismas permite procesar el conocimiento y el intercambio de experiencias, así se pueden aportar nuevos insumos para el reencuentro. Este es un ejercicio constante de ir y venir para poder crecer en el cambio constante.

Tercera idea, "multiplicar-multiplicar". Se multiplica cuando se comparte y se crece en conexión con otras personas que, al mismo tiempo, están autoconectadas y pueden hacerlo con otras. En otras palabras, el intercambio de experiencias es genuino en la medida en que las personas están comprometidas con lo que hacen y quieren transformar con el aporte de otras personas que están en ese mismo proceso. Por esta razón, este modelo multiplicador es efectivo, en primer lugar cuando se trabaja con grupos medianos o pequeños (de 5 a 25 personas); en segundo lugar, cuando se planifica el proceso haciendo uso de un posicionamiento teórico y, en tercer lugar, cuando la intención se dirige al aprendizaje y desarrollo de habilidades específicas con los grupos meta.

La experiencia que se comparte deja un gran aprendizaje y es que, aunque la tendencia de diversos profesionales y madres y padres de familia sea participar en charlas de corte cognitivo, vale la pena darse la oportunidad de ir más allá de las ideas y de arriesgarse a trabajar con las relaciones y la convivencia en familia.

A manera de resumen, la estrategia de "agentes multiplicadores" es de gran utilidad para el trabajo con grupos que se proponen entre sus objetivos profundizar en sus vivencias y mejorarlas. Esas vivencias y los grupos pueden ser muy diferentes, como lo son las familias entre sí; sin embargo, lo que se busca es muy similar cuando se trata de mejorar la convivencia en familia. Este fue el interés de todas las personas participantes, desde el equipo capacitador, hasta quienes participaron en la capacitación y hasta las familias que se beneficiaron. 
URL: http://www.una.ac.cr/educare

CORREO: educare@una.cr

\section{Referencias}

Beavers, W. R. y Hampson, R. B. (1995). Familias exitosas: Evaluación, tratamiento e intervención. Barcelona: Paidós.

Calderón, E., Murillo, J., Villalobos, A., Villanueva, R. y Villarreal, C. (2009). Proyecto Fortalecimiento de la familia. (Informe final 2007-2009, código unidad ejecutora 050804 y presupuestario 031501) Heredia: División de Educación para el Trabajo, CIDE, Universidad Nacional.

Dinkmeyer, D., Sr., Mckay, G. D. y Dinkmeyer, D., Jr. (1998). Guía para los padres. Preparación sistemática para educar bien a los hijos. Estados Unidos: STEP Publisher.

Fuhrmann, I. y Chadwick, M. (1998). Fortalecer la familia. Manual para trabajar con padres (3a ed.). Santiago: Editorial Andrés Bello.

García, N. (2001). La orientación en grupos. En R. Bisquerra (Coord.), Modelos de orientación e intervención psicopedagógica (pp. 341-362) Barcelona: CISSPRAXIS.

López, S. y Escudero, V. (2003). Familia, evaluación e intervención. Madrid: Editorial CCS.

Richardson, R. W. (1993). Vivir feliz en familia. Barcelona: Paidós.

Sanz, R. (2007). Orientación psicopedagógica y calidad educativa. Madrid: Pirámide.

Satir, V. (2005). Nuevas relaciones humanas en el núcleo familiar (2ª ed.). México, DF: Editorial Pax.

Watzlawick, P., Beavin, J. y De Avila, D. (2002). Teoría de la comunicación humana. Barcelona: Editorial Herder.

\section{Cómo citar este artículo en APA:}

Villarreal, C., Villalobos, A. L., Villanueva, R. (enero-abril, 2014). La modalidad de agentes multiplicadores para orientar a madres y padres de familia. Revista Electrónica Educare, 18(1), 219-238. Recuperado de http://www.revistas.una.ac.cr/index.php/EDUCARE/issue/current

Nota: Para citar este artículo en otros sistemas puede consultar el hipervínculo "Como citar el artículo" en la barra derecha de nuestro sitio web:

http://www.revistas.una.ac.cr/index.php/EDUCARE/index 